Article

\title{
Small-scale rotor aeroacoustics for drone propulsion: a review of noise sources and control strategies
}

\author{
Paolo Candeloro ${ }^{1}$, Tiziano Pagliaroli ${ }^{1}$, Daniele Ragni' ${ }^{2}$ and Silvia Di Francesco ${ }^{1,}$ \\ 1 Università Niccolò Cusano, Via Don Carlo Gnocchi 3, 00166 Rome, Italy \\ 2 TUDelft Aerospace Faculty, AWEP Department, Kluyverweg 1, 2629HS, Delft \\ * Correspondence: paolo.candeloro@unicusano.it
}

\begin{abstract}
In the last decade, the drone market has grown rapidly for both civil and military purposes. Due to their versatility, drones demand is constantly increasing, with several industrial players joining the venture to transfer urban mobility to the air. This has exacerbated the problem of noise pollution, mainly due to the relatively lower altitude of these vehicles and to the proximity of their routes to extremely densely populated areas. In particular, both the aerodynamic and aeroacoustic optimization of the propulsive system and of its interaction with the airframe are key aspects of the design of aerial vehicles for the success or the failure of their mission. The industrial challenge involves finding the best performance in terms of loading, efficiency and weight, and, at the same time, the most silent configuration. For this reason, research has focused on an initial localization of the noise sources and, on further analysis, of the noise generation mechanism, focusing particularly on directivity and scattering. The aim of the present study is to review the noise source mechanisms and the state-of-the-art technologies available in literature for its suppression, focusing especially on the fluid-dynamic aspects of low Reynolds numbers of the propulsive system and on the interaction of the propulsive-system flow with the airframe.
\end{abstract}

Keywords: Drones; Aerodynamics; Aeroacoustics; Rotor Noise; Airframe Noise; Porous Material

\section{Introduction}

The term "drone" refers to an automatized vehicle with high manoeuvrability, in both hovering and cruise operations. In the most interesting configurations, Unmanned Aerial Vehicle (UAVs), small multicopter Unmanned Aerial Systems (UAS) or Micro Aerial Vehicle (MAV) are already designed with vertical or horizontal take-off and landing capabilities, and can manoeuvre with extremely high versatility and speed. Due to their unique properties, MAVs are often used in tactical surveillance missions or for reconnaissance purposes. In order to gain information about the scouting area without being easily identified, achieving an acoustically stealth-mode is an essential feature of mission success. Despite the different aims, the noise footprint of these vehicles is extremely important even when employed in civilian roles, due to their flight proximity to populated urban areas. Some of their mission tasks still require geographical mapping, infrastructure inspections, precision agriculture, delivery and e-commerce. Small drones will have an enormous social and economic impact. In fact, this technology opens new possibilities in several application fields. For example, drones equipped with cameras can resolve the problem of the images taken by satellites (which are often expensive, weather-dependant and in low-resolution) or car-based images (which are limited to human-level perspectives and the availability of accessible roads). In addition, farmers can check the quality of crop growth by using cameras mounted on specific UAV. These particular drones will also enable construction companies to verify work advancement in real time. For mining companies, interest focuses on the possibility of obtaining precise volumetric data, leading to lower risks for their employers. Humanitarian 
organizations will be able to evaluate and adapt aid efforts for refugee camps, while medical supplies can be delivered quickly by rescue organizations where necessary. By using MAVs for transportation, developing countries (i.e. countries without appropriate road networks) could deliver goods simply. Inspection drones, vehicles able to fly in confined space, can be used by fire-fighting and emergency units to assess danger faster and safely, or by logistic companies to detect damage to both inner and outer shells of ships, or by road maintenance companies to measure deterioration in bridges or tunnels. Security agencies will be able to improve building safety by monitoring even the areas outside cameras range. Drones will enable disaster mitigation agencies to inspect partially collapsed buildings in the event of obstacles for terrestrial robots. Teams of autonomous drones coordinators will enable missions to last longer than the flight time of a single drone by allowing it to leave the swarm for a short time to replace the battery [1].

An interesting application for drones is their ability to provide accurate surface flow maps of sub-meter water bodies [2,3]. With These vehicles, remote and distributed non-invasive flow measurements can be taken in water environments that are difficult to access. By using drones, on site surveys, which are typically used for traditional measurements, will be unnecessary, allowing for inaccessible area to be observed. Generally, in situ stations provide observation points that are too widely spaced to achieve spatial patterns. On the other hand, UAV can open up several possibilities in land and water monitoring because of low-altitude flight, low cost and flexible payload design [3]. Micro-UAVs can also be employed for analysing large-scale environmental and hydraulic parameters [4]. In particular, they focus on the spatial and temporal extension of reed beds(common reed). The advantages of using UAVs is their extreme portability, easy driving and lower costs and the possibility to fly in dangerous areas reducing enormously risks for the employers. On the contrary, the disadvantages are the limited weight and dimension payload and the instabilies in bad weather conditions. Nevertheless, results show that UAV system can be considered an alternative to the traditional monitoring methods. In fact, UAVs guarantee maps with sufficient accuracy due to their mechanic characteristics and the usability of the control software.

Additionally, the combination of distributed or multi-rotor propulsive systems, generally preferred for manoeuvrability, and proximity to civil areas makes drone noise a challenging issue for the European scientific community at both industrial and academic level. In a 2018 document, the European Aviation Safety Agency (EASA) specified the noise level requirement for drones at a fixed value of $60 \mathrm{~dB}(A)$, measured at a distance of $3-m$ from the source [5]. Generally, the strategic objectives for drone market growth are greater endurance and acoustic impact reduction. These two aspects are also key issues to improve the safety of this technology in the future. Drone noise pollution is also a problem from the point of view of public acceptance of the widespread deployment of flying drones in urban areas. To give an idea of public acceptance of large-scale use of drones in residential areas, information about the effects on the population of a large-scale test drone for delivering can be found in an article from the Wall Street Journal ("Delivery Drones Cheer Shoppers, Annoy Neighbors, Scare Dogs", WSJ 2018 [6]). In this article, drone noise is indicated as the main obstacle to widespread public acceptance of this technology in residential areas. Furthermore, it is known that exposure to aircraft noise might be a significant cause of community reaction and social disturbance. Using a definition of health that includes both mental and social well-being, it is true and a well-known fact that being exposed to aircraft noise causes ill-health. Several studies indicated that aircraft noise exposure can be associated with a prevalence of psychological and psychiatric symptoms. Studies show a strong link between aircraft noise and sleep loss and awakenings [7]. These effects can be a further motivation to find a way to reduce noise generated by UAVs.

Despite the clear drawbacks related to acoustic emissions, drones are earmarked to transform the marketplace of deliveries and civil urban transports, speeding up delivery times and reducing costs, which is what the companies are betting on them [6]. The global drone market will have grown from $\$-2$ billion in 2016 to almost $\$-127$ billion in 2020 [8]. Growth is so fast that this technology is expected to encourage innovations that will disrupt existing industries. In addition, interest in this 
topic can also be seen in the European Union $U$-space project. U-space is a set of new services designed to guarantee safe, efficient and secure access to airspace under $150-m$ for a great number of drones. This would facilitate any kind of routine mission in all classes of airspace and all types of environment. One additional and often overlooked drone applicationis the monitoring and scouting of wildlife. The impact of UAVs on the animal population has been the object of recent research [9]. What the studies have found is that drones constitute a potential new source of anthropogenic disturbance, and depends both on UAV configurations themselves and on additional environmental factors. Mulero-Pazmany et al. [9] suggest that animal reactions are not only influenced by the magnitudes of the noise levels, but also by the sound intermittency and timbre. In the case of a UASs, these changes in intensity may be associated with aircraft on-flight engine variations due to sudden trajectory changes, or due to wind gusts, which has led to the extension of the aeroacoustic problem to unsteady regimes. Noise signature has been additionally addressed as one of the main influencing parameters on both human and animal behaviour [10,11]. Long-term exposure studies based upon the acoustic emissions of UASs have yet to be performed. However, according to recent studies, the physiological and behavioural aspects associated to psycho-acoustic stress [7] are expected to potentially cause relatively higher energy expenditures, decreases in reproduction and survival, and space-use changes, which might compromise the average fitness or even viability of certain populations. Even marine mammals could be negatively affected by UAVs noise emission. In [12,13] drone noise and visual cues are the main problems for the utilization of drones in wildlife science. These situations require drones to fly at close range (less than $10-m$ ), increasing the risk of disturbance for marine mammals. I- air-recording showed that the noise level generated by UAVs (they considered two commonly used drones in marine mammal research) were within the level known to cause disturbance in some animals.

There is interest in this topic from both the academic and industrial spheres. The main manufacturer moving to design a silent configuration is DJI, which designed the Mavic Low Noise Propeller, which seems to reduce noise to almost 60\%, measurable in $4 d B$. In addition, the Master Airscrew has designed a low noise propeller for the DJI Mavic Air that generates low-pitch sound compared to the original props. The new designed propeller reduces the aircraft noise by up to $3.5 \mathrm{~dB}$ and increases the flight time by $12 \%$ which means 2.5 minutes of extra flight time for the standard Mavic Air battery. From an academic point-of-view, different research groups are focusing on UAV noise. The main example are the University of Southampton, the Institut Supérieur de l'Aéronautique et de l'Espace (ISAE-SUPAERO) and Niccolò Cusano University. The university of Southampton is working mainly on leading edge modification to reduce interaction noise $[14,15]$, whereas main research topic of ISAE-SUPAERO is the design of a quiet propeller by means of an optimization process [16]. Instead, Niccolò Cusano University is focusing on trailing edge modification to reduce the broad-band noise component generated by propellers [17]. Furthermore, numbers of research groups working on this topic have increased.

For propeller-driven aircraft, the main noise sources are the engine and the propeller itself, and this problem strongly affects low Reynolds regime too. Therefore, to reduce drone noise signature, the only way to proceed is to optimize both components at the same time. For this reason, in recent years, there has been renewed interest in the first aeronautical propulsion device: the propeller. Rotor noise is becoming a very central issue because of the several fields of drone application. Due to constraints in size and power-density, MAVs are typically equipped with electric motors, which contribute to simplify operations and significantly reduce the mechanical noise signature. As reported in literature, the greater benefit is achieved through the usage of brushless motors [18]. In the last few years, the reduction of noise from the propulsive system of small rotors has been the subject of several works in literature [18-26]. Propeller noise reduction requires particular care in the design process because the achieviement of an aeroacoustic optimum may affect the generation of aerodynamic forces.

While several previous works focused on relatively high Reynolds numbers propeller, few studies have focused on low-Reynolds small-scale propellers. For the latter kind of propellers, especially in hovering conditions, where a considerable area of the rotor is subjected to stall and to self-interaction 
with its own slipstream, the effect of the flow features, such as recirculation bubbles, stall cells and non-uniform boundary layer transition, are exacerbated.

In the Fourier domain, the noise footprint of these propellers exhibits two main noise components: tonal and broadband contributions $[20,27,28]$. While the tonal component is associated with the rotational regime of the blade, the broadband component is due to the convection of flow structure along the leading/trailing edge of the blades. The presence of the aforementioned flow structures, associated with laminar separation or three-imensional spanwise flow non-uniformity contributes to a reduction in effective loading and an increase in the unsteadiness at the blade edge. Small-scale UAVs provide a great challenge to the task of noise characterization and prediction. Indeed, the main noise sources remain consistent with those associated with helicopters, but there are numerous unknowns which could be investigated. For example, the effect of reduced size and the balance between tonal noise and broadband noise. An important difference between small size UAVs and conventional rotorcraft is the flow speed regime in which they fly, measured by the chord-based Reynolds number at $75 \%$ span:

$$
R e_{c}=\frac{0.75 R \rho_{\infty} \Omega c}{\mu_{\infty}}
$$

where $\Omega$ is the rotational regime, $R$ is the rotor tip radius, $\rho_{\infty}$ is the air density, $c$ is the rotor blade chord and $\mu_{\infty}$ is the air dynamic viscosity. For a full-scale helicopter, a representative $R e_{c}$ is in the order of $10^{6}$, while for a UAV it may range from $10^{4}$ to $10^{5}$. In terms of conventional flat plate aerodynamics, the former Reynolds number explicates in a turbulent flow regime while the latter in a laminar-transitional flow regime [29]. This discrepancy calls into question the applicability of traditional noise prediction tools.

When summarizing the different contributions, literature shows that broad-band contributions in the noise footprint can be due to: incoming-flow turbulence at the blade leading edge (i.e. LE noise from highly turbulent flow in harsh environments), interaction of the boundary layer with the blade trailing edge (i.e TE noise due to turbulent boundary-layers but also due to unsteady flow separation of re-circulation bubbles etc), flow separation of the flow on the different blade sections (i.e. stall and flow separation noise), blade vortex interaction (i.e. BVI due to the interaction of a rotor blade with the shed tip vortices from a previous blade) [20]. Predicting and reducing the noise radiation from these contributions is even more complicated due to the variety and sensitivity of the noise to the flow field. These reasons clarify the complexity of the problem and the importance of improving knowledge in this field.

In literature, a few studies were devoted to the analysis of the noise due to the interaction between the propulsive system and the airframe in the case of small propeller. Zawodny et al. [30] in their experimental analysis found that the presence of airframe surfaces is a not-negligible noise source. In fact, it generates noise levels analogous or even greater than the rotor blade surfaces in particular rotor tip conditions. This study analyzed the effects of both airframe to rotor distance and airframe size. Results show prominent tonal peaks in the Fourier domain related to airframes in the case of close proximity between the airframe and the rotor plane. This effect seems to decay rapidly if the rotor-airframe distance increases. Even, the airframe shape seems to influence noise generation. Generic constant cross-section systems were found not to affect noise generation in the plane of the rotor. Instead, a conical airframe shows an increase in the tonal noise component.

The manuscript is organized as follows. In §2.1 there is a brief explanation of the most important noise sources for rotors, $\$ 2.2$ reviews the state of art of passive control strategies currently in use. Finally, §3 draws conclusions and provides a brief overview of future configurations.

\section{Noise in Drones}

This section provides a brief explanation of the most common noise prediction model and a list of the most interesting passive noise control strategies found in literature. The aim is to understand 


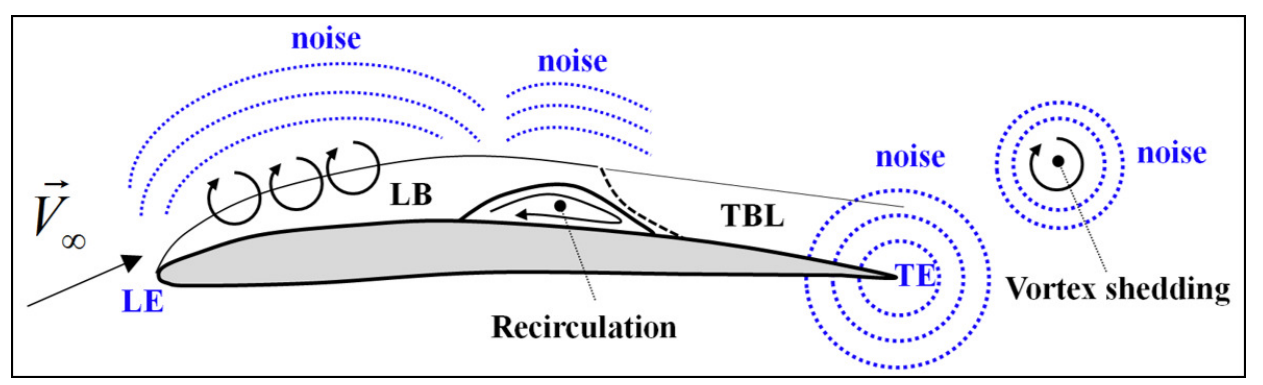

Figure 1. Representation of the main noise sources around an airfoil.

the noise generation mechanism and how this phenomenon can be mitigated. The main problem is to guarantee the aerodynamic performance necessary for proper mission development.

\subsection{Noise modelling: tonal and broad-band}

The aerodynamic noise of conventional propellers can be split into two main components in the Fourier domain: tonal and broad-band contributions [20,28].

Tonal components are directly related to the periodic motion of the blade in the surrounding fluid. Therefore, the frequency and magnitude of the radiated noise is related to rotational velocity. The physical mechanism associated with the production of the tonal contributions is related to blade thickness and to aerodynamic loading.

On the other hand, broad-band noise is radiated by the interaction of turbulent flow structures with the blade edge. Therefore, it is either generated at the blade leading/trailing edge or at the blade tip. Research studies tend to separate pressure fluctuations, denoted as $p^{\prime}$, radiated from the blade surface in the far field, into two components $[20,27,28]$ :

$$
p^{\prime}=p_{N B}^{\prime}+p_{B B}^{\prime}
$$

Where $p_{N B}^{\prime}$ is the narrow-band component of pressure fluctuations, whereas $p_{B B}^{\prime}$ is the broad-band counterpart. The theoretical prediction of the periodic noise generated by propellers is based on the solution of the Ffowcs, Williams and Hawkings non-homogeneous wave equation, known as the Ffowcs-Williams/Hawkings equation [21,31].

$$
\frac{1}{a^{2}} \cdot \frac{\partial^{2}\left(p^{\prime}\right)}{\partial t^{2}}-\frac{\partial^{2}\left(p^{\prime}\right)}{\partial x_{i}^{2}}=\frac{\partial^{2} T_{i j}}{\partial x_{i} \cdot \partial x_{i}}+\frac{\partial}{\partial t}\left\{\rho_{a} \cdot v_{i} \cdot \delta(f) \cdot \frac{\partial f}{\partial x_{i}}\right\}-\nabla\left\{\Delta p_{i j} \cdot \delta(f) \cdot \frac{\partial f}{\partial x_{i}}\right\}
$$

where $a$ is the speed of sound, $\rho$ is the air density, $p^{\prime}$ is the perturbation on the static pressure, $t$ is the observer time and $x_{i}$ are the components of the position vector, $T_{i j}$ are the components of the Lighthill stress tensor, $p_{i j}$ are the components of the generalized stress tensor, $v_{i}$ the components of the source velocity vector, $\delta$ is the Kronecker's delta function and $f$ is a function that defines the surface of the body producing the pressure wave.

In this equation, there are 3 forcing terms on the right-hand side which are related to vortex,thickness and loading. For thin blades and low Mach numbers $(M<1)$, the vortex term is negligible and the narrow-band contribution is given by the sum of a sound source related to blade thickness $p_{T}^{\prime}$ and one related to aerodynamic loading $p_{L^{\prime}}^{\prime}$ as distributed force over the blade:

$$
p_{N B}^{\prime}(\mathbf{x}, t)=p_{T}^{\prime}(\mathbf{x}, t)+p_{L}^{\prime}(\mathbf{x}, t)
$$

The thickness term takes into account the fluid displacement due to the body, while the loading counterpart takes count of the unsteady force distribution over the body surface.

A numerical evaluation of these two quantities can be achieved by discretizing the blade in $N$ finite 
elements along the span. The resulting overall radiation field is approximated as the sum of $N$ pointwise sources.

$$
\begin{aligned}
& p_{L}^{\prime}(\mathbf{x}, t)=\sum_{k=1}^{N} p_{l, k}(\mathbf{x}, t) \\
& p_{T}^{\prime}(\mathbf{x}, t)=\sum_{k=1}^{N} p_{t, k}(\mathbf{x}, t)
\end{aligned}
$$

Using a reference system of coordinates $\mathbf{x}=(x, y, z)$ as defined in Fig.2, the two components can be calculated using Eqs. 7-8 (see [20]), which are derived in [28,32]:

$$
\begin{gathered}
p_{L, k}^{\prime}(\mathbf{x}, t)=\frac{1}{4 \pi}\left\{\frac{\dot{\mathbf{F}}_{k} \cdot \hat{\mathbf{r}}_{\mathbf{k}}+\mathbf{F}_{k} \cdot \hat{\mathbf{r}}_{k}\left[\frac{\left(\dot{\mathbf{M}}_{k} \cdot \hat{\mathbf{r}}_{k}\right)}{\left(1-M_{r_{k}}\right)}\right]}{a r_{k}\left(1-M_{r}\right)^{2}}+\frac{\mathbf{F}_{\mathbf{k}} \cdot \hat{\mathbf{r}}_{k}\left[\frac{\left(1-\mathbf{M}_{\mathbf{k}} \cdot \mathbf{M}_{\mathbf{k}}\right)}{\left(1-M_{r}\right)}\right]-\mathbf{F}_{\mathbf{k}} \cdot \mathbf{M}_{\mathbf{k}}}{r_{k}^{2}\left(1-M_{r}\right)^{2}}\right\} \\
p_{T, k}^{\prime}(\mathbf{x}, t)=\frac{\rho}{4 \pi} \frac{\partial}{\partial^{2} \tau^{2}}\left\{\frac{\Phi_{k}}{r_{k}\left(1-M_{r}\right)}\right\}^{2}
\end{gathered}
$$

where $\hat{\mathbf{r}}_{\mathbf{k}}$ is the position vector of an observer relative to the $k$-point noise source $(|\hat{r}|=1), \mathbf{F}_{\mathbf{k}}$ is the aerodynamic force on the $k$-point blade element of volume $\Phi_{k}$. The Mach vector is defined as $\mathbf{M}_{\mathbf{k}}=\frac{\mathbf{v}}{a}$ and the scalar magnitude $M_{r_{k}}$ represents the component of $\mathbf{M}_{\mathbf{k}}$ on $\mathbf{r}_{\mathbf{k}}$. If $t$ is time as measured in the observer's reference frame, retarded time $\tau$ indicates the time when the pressure wave left the noise source. Observer time $t$ and retarded time $\tau$ are connected by:

$$
\tau=t-\frac{r(\tau)}{a}
$$

In Eq.7, the first term represents the far field, while the second is representative of near field contribution. These two terms differ by the power of $r_{k}$ in the denominator. The far-field term is proportional to $r_{k}^{-1}$ while the near field term is proportional to $r_{k}^{-2}$, thus the last term becomes relatively small at large distances from the noise sources [21].

On the other hand, the broad-band noise of a propeller is generally produced by three main sources: noise related to the turbulence of the incoming flow ( $L E$ noise), noise produced by the interaction of the turbulent boundary layer over the blade surface with the trailing edge (TE noise) noise generated by the possible separation of the flow (Separation noise) [20]. Therefore, the broad-band contribution can be further split as:

$$
p_{B B}^{\prime}=p_{T E}^{\prime}+p_{L E}^{\prime}+p_{S}^{\prime}
$$

where $p_{T E}^{\prime}$ is the TE component, $p_{L E}^{\prime}$ is the LE component and $p_{S}^{\prime}$ is the separation term.

Several authors have addressed the prediction of trailing edge broad-band noise in literature. A relation between the Power Spectral Density of the trailing noise $\left(S_{p p}^{T E}(r, \theta, \omega)\right)$ and the spanwise velocity correlation length $l_{y}$ is reported in [20] as:

$$
S_{p p}^{T E}(r, \theta, \omega)=\frac{B}{8 \pi}\left(\frac{\omega c}{2 a r}\right)^{2} \Delta R D(\theta, \phi)|I|^{2} \Phi_{p p} l_{y}
$$

where $c$ is the chord, $\Delta R$ is the spanwise length of the blade, $I$ is the radiation integral function, $B$ is the number of the blades, $\omega=2 \pi f$ is the angular frequency, $f$ is the rotational frequency, $D(\theta, \varphi)$ is the directivity function and $\Phi_{p p}$ is the wall power spectral density of the pressure fluctuations. The wall pressure spectral density $S_{p p}^{T E}$ and the spanwise correlation length $l_{y}$ can be evaluated experimentally or numerically. There are different models for $S_{p p}^{T E}$ estimation, e.g. the one proposed by Schklinker and Amiet [33], or the more recent model proposed by Rozenberg et al. [34], which takes into account the effect of the adverse pressure gradient. On the other hand, for $l_{y}$ evaluation the most used model is the Corcos' model [35]. 

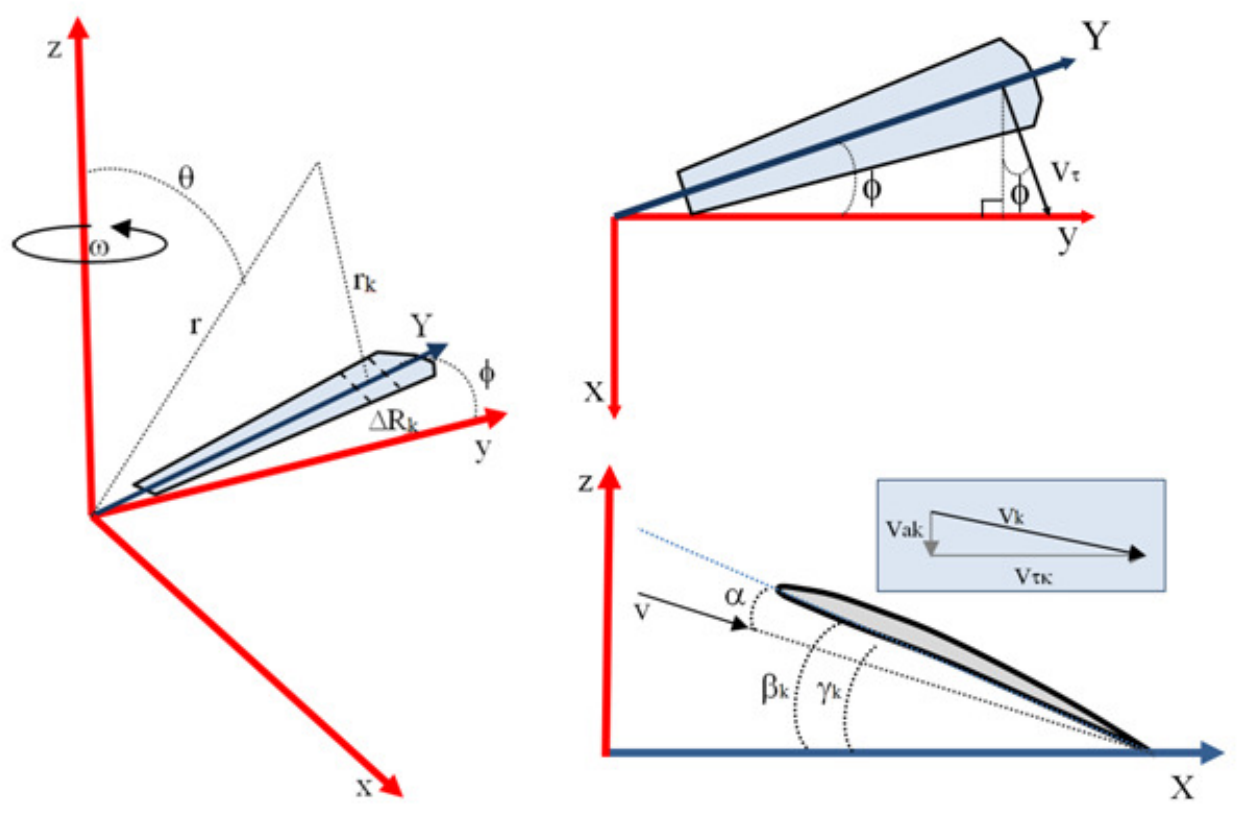

Figure 2. Representation of the reference coordinate system considered for the definition of the aeroacoustic model.

The effect of the flow separation on broad-band noise can be significant as well. According to [25], an estimation of the power spectral density is provided by the following expression:

$$
S_{p p}^{s e p}(\mathbf{x}, \omega)=\left(\frac{\omega}{4 \pi a r}\right)^{2}\left(\rho^{2} c U^{2} A_{S}^{2}\right)\left(\frac{z}{r}\right)^{2}\left\{\frac{c_{D}^{2}}{4}\left[\frac{2 \pi U}{\omega c\left(1-M_{r}\right)}\right]^{3} 8.6 \cdot 10^{-7}\right\}
$$

where $c_{D}$ is the drag coefficient, $A_{S}$ is the body cross-sectional area where separation is localized and $U$ is the velocity of the flow.

\subsection{Noise reduction strategies}

As pointed out in the previous section, UAV and MAV propeller noise is a central and complicated issue that has to be taken into account in system design. This section describes the most effective noise control techniques, especially the physical mechanism that enables noise reduction and the changes in aerodynamic performance induced by the noise control system itself.

There are Two basic strategies to control the noise generated: active and passive. Large scale airfoils and propellers have employed active flow control methods, but these solutions require energy expenditure. These methods include active modifications of airfoil geometry or of flow conditions, which is achieved by either modifying airfoil geometry and surface through actuators, or by acting on the local boundary layer through blowing and suction jets. Due to the typical sizes of the control systems and of the actuators, these technologies are not suitable for small-scale propellers employed by MAVs. On the other hand, passive flow control techniques enable the boundary layer to be manipulated without further consumption of additional energy, and it can be employed to reduce noise generation. For this reason, there have been several studies on them in the last decade [36]. Consequently, in this paper the focus is on the second control method. The passive control methods employed to reduce noise generation include serrations, porous materials application, boundary layer tripping and geometry 
optimization. The use of serrations is of particular interest in this work due to its potential noise reduction efficiency.

\subsubsection{Optimized Geometry}

The general aim of the propeller design process is to find the best aerodynamic performance without considering aeroacoustic behaviour. This is achieved by means of an optimization-based design process. Optimization theory points out that an optimal design problem can be described mathematically by looking for a configuration that minimizes (or maximizes) a certain cost function $J$ that embodies the design objective [21]. For rotors, most of the existing methods are based on the work of Betz [37] from 1919. This approach focuses on finding the optimal propeller geometry in order to minimize the power required to obtain a certain propulsive force (or to maximize the thrust produced by a certain power) at a certain specific operating condition (which has to be interpreted as a combination of airspeed, altitude, and propeller rotational speed). To design a quiet propeller, acoustic requirements must be included and an iterative process was commonly employed. First, the optimal propeller in aerodynamic terms is defined (i.e. with maximum efficiency). Then, the resulting propeller is further modified in order to improve its acoustic properties [28,38,39]. This is the "classical" procedure for quiet propeller design, but such an iterative process presents some complications. It does not ensure an optimal final design and it is also difficult to introduce additional constraints into this serial design process, such as side or structural constraints. An improvement of this process is to implement a multidisciplinary design optimization (MDO) approach [40,41]. MDO ensures that all the different disciplines are addressed simultaneously. In this case of study, aerodynamic, structural and acoustic problems were analysed at the same time.

One of the most interesting MDO models in literature was presented by Gur and Rosen $[18,21,42,43]$, developed to reach the best compromise between the opposite requirements of efficiency and quietness. In particular, the target of this design process is first to mitigate the tonal component of the noise, dependent on the actual loading of the blade. In [21], the cost function $J$ is based on the Sound Pressure Level (SPL). However, the presence of power and stress constrains were taken into account. The first step was to optimize only the acoustic footprint of the blade, and interestingly, MDO results provides for a blade with a very large chord and relatively small radius. This is, of course, unfeasible in a small-rotor, due to the power required by such a non-optimal aerodynamic design in combination with the noise increase from the electric engine to deliver such a power at the hub. Furthermore, a limit on the extracted power from the battery produces a significant increase in propeller noise. Instead, stress constrains lead to an increase in cross-sectional thickness and rotational speed. These results clarify the need for a multidisciplinary optimization. In fact, the presence of both structural and acoustic constrains is fundamental to achieve feasible results. This model was enhanced in [18], where the propeller design model was extended to the entire propulsion system. In other words, a model for electric motor and battery was added to the previous model. For this purpose, theoretical models of these components are required. The models presented are based on a comprehensive investigation of existing motors and batteries. The performance of the vehicle greatly depends on the interaction between propeller, electric motor and battery. Clearly, then, it is important to study these three components contemporaneously.

By using Gur and Rosen model [21], Sinibaldi and Marino [20] employed a quiet propeller and carried out an experimental analysis to characterize its behaviour as compared to a conventional one (conventional in the sense of a propeller not specifically designed to achieve noise reduction). In their study, the focus is on the optimization of the chord distribution along the span-wise direction. The results of the comparison of the two propeller show that, by using the MDO approach, significant noise reduction can be achieved, at least for the narrow-band contribution. An unexpected result is that by increasing rotational velocity, in order to achieve high thrust values, strong vibrations occur that can be ascribed to the increased thickness of the optimized blade. This phenomenon produces noise that make the optimized propeller comparable with the conventional one. 
Pagliaroli et al. [19] used an MDO approach in order to assess the effect of the pitch angle on MAV noise signature. An experimental analysis was carried out in order to evaluate aeroacoustic behaviour. The experimental tests were carried out on a propeller that has $2[\mathrm{~mm}]$ and a twist angle of zero. The blades are mounted on a collective pitch in order to vary the pitch angle from 0 to 21 [deg]. All the measurements were taken at the anechoic chamber of the Office National d'Études et de Recherches Aérospatiales (ONERA). The optimization strategy seems to be useful in reducing the number of variables in the multiphysics problem. Furthermore, wall pressure measurements confirm that the pressure signature is dominated by the broad-band component generated by the separation bubble, showing that it is important to extend studies to broadband noise.

\subsubsection{Serrated Trailing Edge}

In literature, one of the most interesting and investigated noise control strategies is based on the application of serrated trailing edges (STE). Serrations applied to the TE of an airfoil reduce noise generation due to the destructive interference of the pressure fluctuations produced by the flow structures convecting along the slanted edge. This technique is already employed on wind turbine blades and fixed wing airfoil. Nevertheless, there have been a few studies on the application of serrations to small rotors. Fig.3(b) shows a representation of a blade with the serration at the TE. The idea for this control strategy was inspired by nature, in particular by the silent flight of owls [44-46]. Owls are known to be one of the most silent predators in nature. The quietness of their flight is due to their characteristic wings, with three main physical features: ae suction wing surface with a soft downy coating; a comb of stiff feathers at the wing leading edge, and TE feathers and wings with a fringe of flexible filaments. The sawtooth pattern employed by manufacturers is the simplest geometric way to mimic the permeability of owls' wings.

Chong et al. [47] and Avallone et al. [48] focused on wind turbine applications. The first study involved an experimental analysis on a flat plate, while the second was a numerical investigation on an airfoil at zero degree angles of attack by resolving the Ffowcs-Williams/Hawkings analogy (see §2.1). On the basis of Howe[49], Chong et al., pointed out that significant noise reduction can be achieved if two conditions are met. The first is that the serration length is of the same order of the turbulent boundary layer thickness $\delta$ near the TE. The second is that the serration angle (called $\alpha$ in Fig.4) is small, favouring sharp sawtooths. Howe's theoretical approach states that the introduction of obliqueness at the TE will reduce the coherence between the acoustic sources along the wetted surface. This effect will result in weaker noise emission. The experimental acoustic results show that TE broad-band noise can be significantly reduced by using serration. Furthermore, noise reduction has been found to occur in a large range of frequency. In [47], hot wire anemometry (HWA) measurements are aimed at determining coherent structures on a flat plate surface. The measurements show that wake structures are affected by serration since noise reduction can be ascribed to this phenomenon. On the other hand, Avallone et al.[48] investigate the physical noise reduction mechanism by means of a numerical simulation based on lattice Boltzmann and Ffowcs-Williams/Hawkings equations (see §2.1). The propeller analysed is a "conventional" sawtooth and a combed-sawtooth TE. For the combed-sawtooth geometry, the space between the teeth was filled with solid filaments called combs (see Fig.4). Noise reduction was found to depend on frequency. Once a critical value was reached, corresponding to $S t_{c}<30\left(S t_{c}\right.$ is the Strouhal number based on the airfoil chord and the free-stream velocity), no noise reduction was observed. For a given serration geometry, the introduction of combs does not modify the frequency range over which noise reduction can be observed but only on maximum noise reduction. Flow fields analysis shows that the introduction of sawtooth serrations promotes the constitution of elongated coherent structures in the wake in the space between two consecutive teeth, together with hairpin vortices along the sawtooth edges. The effect of this modification on the time-averaged flow field is to mitigate both the outer (namely from the centre line toward the edge) and the inner (namely from the edge toward the centre line) flow motions.

Pang et al.[36] involved an experimental analysis of pitch angle and trailing edge serration effect on 
a small rotor. Results show that sawtooth serrations employed at the TE can noticeably suppress broad-band noise in the high frequency region in the far-field. The main drawback observed is that the tonal component seems to increase in the low frequency region. At low velocities, serrations seem to lead to greater noise reduction. Such an effect suggests that STE is a potential solution for reducing UAV noise when propeller are sure to operate at low speed. Near-field experiments shed light on sound field characteristics, exhibiting a radial decay of SPL in the propeller rotation plane.

Ning et al.[50] also carried out an experimental analysis on STE, the aim being to reduce noise while maintaining the thrust constant. This work defines three parameters that ensure a beneficial employment of serration for noise reduction. The parameters considered are:

- the non-dimensional tooth height defined as the ratio between the tooth half-height and the boundary layer thickness $h^{*}=h / 2 \delta$;

- the Aspect Ratio of the tooth defined as the ratio between the width and the half-height $A R_{t}=$ $2 b / h$;

- the boundary layer thickness based Strouhal number $S t_{\delta}=f \delta / U$.

The geometrical parameters employed are defined in Fig.4. Ning pointed out that to achieve noise reduction $h^{*}>0.25$. Otherwise the amplitude of the serration is too small, as a results of which the turbulent eddies go beyond the sawtooth without significant interaction. Furthermore, inclination angle $\alpha$ (see Fig.4) must be lower than $45^{\circ}$ and this fact is guaranteed by imposing $A R_{t}<4$. In the definition of the Strouhal number, $f$ is the sound frequency, $\delta$ is the boundary layer thickness and $U=0.7 * U_{r e l}$ by having called $U_{r e l}$ the relative velocity. This non-dimensional coefficient had to be greater than 1 (as stated in Howe's theory), which means $f>U / \delta$, in order to obtain a significant noise reduction. Experiments have been carried out at $\operatorname{Re}>1.5 \times 10^{5}$. The results show that when $f>U / \delta$, noise reduction appeara at a frequency lower than $U / \delta$, while the overall noise level increases. Therefore, this parameter gives the frequency range in which it is possible to find noise reduction. This work analyses four rotors by varying the $A R_{t}$ coefficient. The analysis involves aerodynamic and aeroacoustic measurements to characterize wake flow statistics. The results show that the STE can reduce broad-band noise in the high frequency region without any loss in aerodynamic performance, while, in the low frequency region, the noise generated is almost the same. Measurements also show that, in order to keep the thrust constant, a higher rotational velocity of the propeller is required.

Also Intravartolo et al.[27] carried out an experimental analysis on STE by focusing on the serration depth effect. Results show that an increase in serration depth produces a reduction in the intensity of the trailing edge wake. Nevertheless, benefits from the depth of the serrations diminished with respect to the overall noise signature of the propeller. When serration depth reaches a value comparable to half of the Mean Aerodynamic Chord (MAC), no further gain in aeroacoustic effect can be observed. On the contrary, an increase in the overall noise may occur due mainly to aerodynamic effects. Serration depth effect is also analysed by Pagliaroli et al.[17], in particular, as regards broad-band noise component and the directivity of the noise source in the near-field. A notable reduction in the noise generated was obtained in the low frequency region and damping in the tails of the probability density function (PDF) was observed. The statistical analysis shed light on the physical phenomenon that lies behind the noise reduction. It is known that PDF's tails are related to intermittent structures in the pressure field, so the serration seems to eliminate strong energetic events. The drawback of serration is a loss in aerodynamic efficiency, so the optimal geometry had to be found. An analysis of the directivity shows that the sawtooth pattern effect is bounded in the polar angle range $\theta=\left[60^{\circ}: 120^{\circ}\right]$ (the polar angle considered is defined in Fig.5).

An improvement to STE technology could be made by the employment of fractal trailing edge geometry. [51] investigates this kind of solution by comparing the behaviours of a sawtooth TE and a conventional TE. An experimental analysis was carried out to test these different TE geometry applied to a flat plate. Noise measurements show that both the sawtooth and the fractal trailing edge produce a reduction in the broad-band noise but an increase in the tonal noise radiated by the tip vortex in the serrations gaps. 


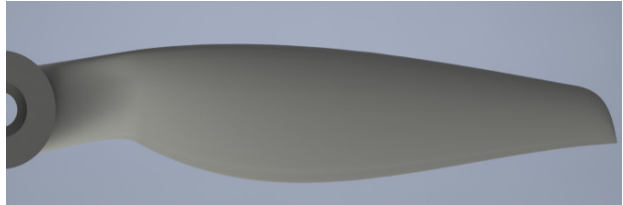

(a)

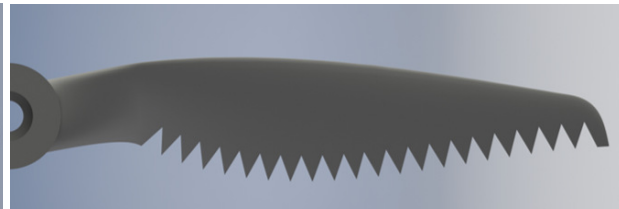

(b)

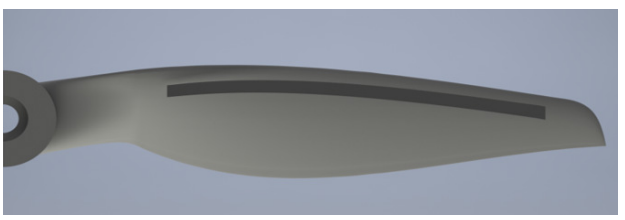

(c)

Figure 3. 3D rendering of the propeller blade: (a) baseline; (b) serrated trailing edge; (c) boundary layer tripping system.
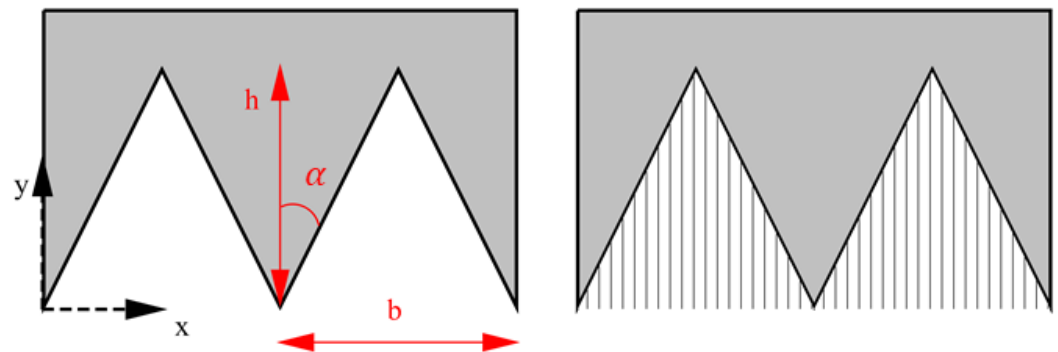

Figure 4. Schematic of the sawtooth and the combed-sawtooth TE geometry analyzed by Avallone et al. [48]

However, the tonal component seems to be mitigated by the fractal TE. This effect may be ascribed to the cancellation of vortex shedding. The investigation on the coherence behind the TE shows that the fractal geometry interacts with the strong coherent structures that always occur between the tips of the serrated TE, by decreasing their strength and extension in all direction. Moreover, the use of sawtooth and fractal-sawtooth TE improved post-stall lift behaviour. Although, lift slightly decreased at some pre-stall angles of attack, the drag did not react significantly to TE replacement.

A mathematical and physical interpretation may be given to the effect of the serrated trailing edges on the noise generated by the propeller. In particular, the serration effect creates destructive interference in pressure fluctuations which are convected along the geometry. From a recent investigations, presented in $[48,52]$, it was found that the frequency spectrum and the boundary layer characteristics develop at the serration edge. This means that the assumption of the theory of "frozen turbulence" cannot be used to analyse of the noise reduction performance. Some of the latest studies $[48,53]$ show that changes in the skin-friction coefficient along the serrations are related to the change of the frequency spectrum and could be used to obtain a more accurate prediction of their response.

\subsubsection{Boundary layer Tripping System}

The experiments of Leslie et al. [24,54] show that broad-band propeller noise emission of a propeller can be reduced by employing a LE boundary layer tripping system on the suction surface of the blade, with negligible evidence of any aerodynamic performance loss. The control technique presented in these works look at a boundary layer tripping system in the form of a simple strip of aluminium adhesive tape. A rendering of the blade with the tripping system is detailed in 8(c). The noise reduction mechanism is related to the mitigation of BL noise because of a forced 


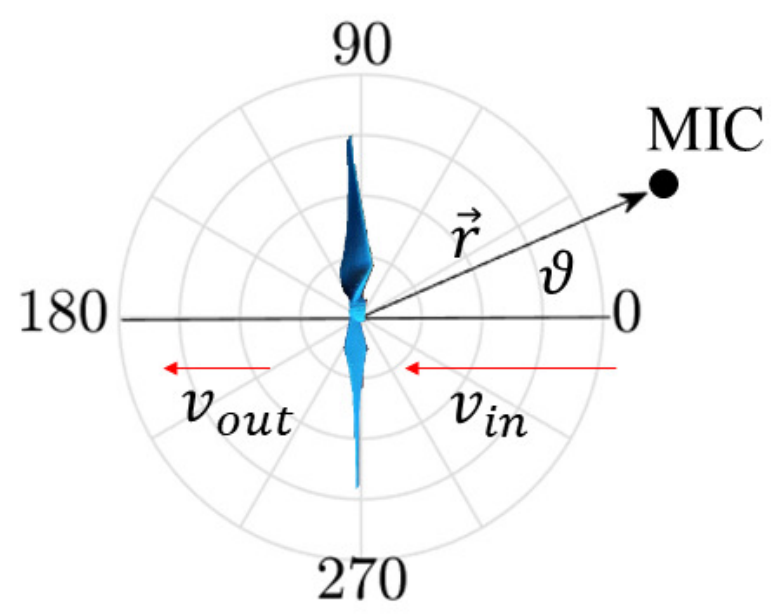

Figure 5. Definition of the polar reference system for the directivity analysis.

laminar to turbulent transition of the BL. Noise generated by the turbulent boundary layer (TBL) is different from the laminar boundary layer (LBL). The LBL generates strong and loud tonal noise, so it appears as narrow-band peaks in the frequency domain. This is the result of an aeroacoustic feedback loop between LBL oscillation and the noise radiated by the TE at the same frequency. Furthermore, the presence of a small laminar separation bubble just prior to the TE helped to amplify the Tollmien-Schlichting (T-S) boundary layer waves, confirming what was found in [55]. In this situation, the presence of the aeroacoustic feedback loop combined with the amplification of the T-S resulted in the production of strong narrow-band tones. By forcing the transition from laminar to turbulent through the use of a transition strip the aeroacoustic feedback loop is broken. The tripping system translates the transition from $80 \%$ to $5 \%$ of the chord and replace the tonal noise with a broad-band noise radiating from the TE. As a result of transition, due either to the presence of a laminar separation bubble, or to forced transition through the use of a transition strip, a TBL is present at the TE of the airfoil. TBL-TE is strongly dependent upon the BL thickness $\delta$ at the TE. The location of the transition affects the TBL-TE noise. If the transition occurs further downstream along the chord, there are smaller contributions from low-frequencies and increased high frequency contributions. Consequently, the tonal noise connected with these two phenomena seems to be mitigated. This passive control technique seems very interesting because it should not affect the aerodynamic properties of the propeller, but rather reduce the drag force.

\subsubsection{Porous materials inserts}

The idea of using porous materials to obtain noise attenuation dates back to the studies of Graham [56] on the silent flight of the owl. Since then, porous materials were added to the blade leading edge in order to reduce noise generation due to strong blade vortex interaction (BVI) in helicopter applications [57]. Another solution is to treat the flap side-edge of the wing with porous material in order to mitigate flap-noise [58]. Recently, porous material has been tested on blunt bodies, such as the cylinder [59], and on flat plat to see if it is possible to reduce noise emission by using them. Another approach previously discussed in literature is the usage of fully porous airfoil [60,61]. These airfoil have a prevalently rough surface. Thus, the drag force generated is expected to increase while the lift force is expected to decrease with respect to the baseline airfoil. Nevertheless, aerodynamic measurements [61] show that there is more lift and less drag as porous material flow resistivity increases. Such a simple dependence cannot be found for the acoustic properties. On the other hand, Geyer et al [60] found that the SPL generated at the TE of the porous airfoil was lower as regard the baseline airfoil for a large range of medium frequencies. Instead, for very high frequency, the porous airfoil has a higher noise signature 
than the non-porous one. As expected, a TBL analysis shows that the porous airfoil has a boundary layer thickness $\delta$ and a displacement thickness $\delta^{*}$ that exceed the non-porous one in both the suction and pressure side of the airfoil.

In recent years, additive manufacturing technology (i.e. 3D printing) has grown very fast and has now made possible to directly integrate porous material structures into airfoil and rotor blade. Jiang et al. [62] carried out an experimental analysis on the effect on the TE noise of porosity employed on a rotor rig. Porous materials have already been used to control flat plate noise generation $[46,47,63]$. In this work, instead, the focus is on a modified propeller with the insertion of a blade extension realized by additive manufacturing. This technology allows designers to employ complex geometries at the TE in order to develop a quiet propeller. The way to attain acoustic stealth is to disrupt the conversion of TBL pressure fluctuations into acoustic waves and reduce the turbulent length scales in the BL, without generating higher levels of turbulence (noise) at unwanted frequencies. Two sets of experiments and one numerical simulation were performed. The first set of experiments involved measuring the acoustic impedance of various additively manufactured samples in order to understand the porous structurs effect on absorption. After this characterization, the porous structures were applied as blade extensions to the outer part of the rotor blade without increasing the rotor diameter. Fig. 6 shows a schematic representation of the blade extension. The experimental results are very interesting, indicating significant noise reduction in the frequency region [1:7] $\mathrm{kHz}$. Numerical simulation showed that the porous TE did not affect the flow field or the BL thickness $\delta$ at the TE. Consequently, the noise reduction observed in the rotor tests may be attributed more to a reduction in turbulence length scale than a disruption of the edge scattering process.

In literature, several authors have studied the effect of porosity on trailing edge noise [64-66]. Rubio Carpio et al. [64] focused on a flat plate with different types of inserts. The porous inserts, covering $20 \%$ of the chord, are manufactured with metal foams of cell diameters $d_{c}=450[\mu \mathrm{m}]$ and $d_{c}=800[\mu \mathrm{m}]$ and permeability values of $6 \times 10^{-10}$ and $2.7 \times 10^{-9}\left[\mathrm{~m}^{2}\right]$. The far-field measurements show low frequency noise attenuation of up to 7 and $11[d B]$, respectively, for the first and second permeability value. On the other hand, in the high frequency region, an increase in noise up to $8-10[d B]$ was observed, this phenomenon is due to surface roughness. By increasing permeability also led to a reduction of the frequency range affected by noise attenuation. A PIV measurement campaign shows an increase in BL thickness $\delta$ and in displacement thickness $\delta^{*}$ for the metal foam insert with higher permeability. Analysis in the Fourier domain shows that the attenuation in velocity fluctuations affects mostly the low frequency region, suggesting that turbulence intensity reduction may be one of the changes that contributes to noise reduction. On the other hand, the results do not show an increase in high frequency fluctuations content as regards the solid case. Showkat Ali et al. [65] demonstrated that porous TE can delay vortex shedding and significantly increase vortex formation length, leading to a very low turbulent near-wake region. The usage of porous material also leads to significant lateral coherence reduction of the turbulent structure.

[66] documents acoustic test on airfoil with porous treatment at the TE. Maximum noise reduction reached was up to $2-6 \mathrm{~dB}$. This effect may be attributed to a material-dependent pressure field generated in the near-field related to the flow resistivity of the TE material.

A very interesting control technique is the use of Poro-Serrated TE [67-69], which combines the serrated TE (\$2.2.2) and the effect of porosity. These poro-serrated TE devices contain porous materials of various air flow resistances at the gaps between adjacent members of the serrated sawtooth. The object of this study is to understand if two control strategies for noise mitigation can co-exist, one related to the oblique edges introduced by the serrations, the second arising from porosity, which allows the pressure side and suction side to communicate, thereby reducing the acoustic dipole strength at the trailing edge. In these studies the focus is on a flat plate with serrated trailing edge with the addition of porous foam between adjacent members of the sawtooth. The porous foam is cut in order to match perfectly with the volume and shape of the sawtooth gaps, thus preserving the original airfoil profile. This technique can simultaneously suppress vortex shedding and reduce broad-band noise. Results 


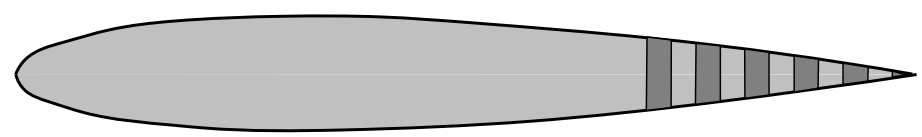

Figure 6. Schematitazion of the section of the rotor extension.

show that multiple broadband noise reduction mechanisms occur (serration + porous material), but it is likely that the porous material is enhancing the serration effect, rather than the porous material exerting an effect of its own.

\subsubsection{Metamaterials}

One way of achieving sound attenuation is the application of sound barrier that reflects or absorbs the incident acoustic energy. Such a solution cannot be directly applied to MAVs because it eliminates the passage of air. To be employed on a rotor blade, it is important to guarantee permeability to air by designing a ducted propeller. In recent years, there has been a fast growth in metamaterial science, leading to new solutions for manipulating acoustic energy. Metamaterials are composed of subwavelength structures since their effective acoustic properties are governed by their structural shape rather than their constitutive properties.

Ghaffarivardavagh et al. [70] present a design methodology for an Ultra-Open Metamaterial (UOM) composed of subwavelength unit-cell structures featuring a predominately open area. The designed UOM works as a high-performance selective sound silencer for applications where both sound attenuation and highly efficient ventilation are required. The proposed method is based on Fano-like interference [71] for selective attenuation of acoustic waves. The first part of their studies aimed to analytically demonstrate that Fano-like interference is present in a transverse bilayer metamaterial. Then, the feasibility of the metamaterial structure was proved by providing both analytic and experimental validation. The designed UOM consist of two distinguishable regions: a central open part and a peripheral helical part. The two regions are characterized by different acoustic properties. Fig.7 reports a 3D representation of it. The contrast in the acoustic properties of the two regions has been proved to be essential to achieve the required silencing functionality. The experimental tests show a reduction in transmitted acoustic energy up to $94 \%$. Another interesting feature of this solution is that the design is inherently flexible. Desired acoustic and refractive index impedance can be achieved by adjusting some geometrical parameters. This feature gives the designer a large number of degrees of freedom in order to optimize device performance.

\subsubsection{Bio-inspired blade Shape}

An innovative bio-inspired UAV propeller is investigated in Ning. [72]. By taking idea from nature and designing a propeller with the planform shape based on cicada wings and maple seeds (see Fig.8(b)). In order to compare it to a conventional propeller (Fig.8(a)), the designed propeller was given the same planform area, the same cross sectional shape, andsame weight. Both the propellers were realized by using additive manufacturing. An experimental analysis was carried out in order to characterize the aerodynamic and aerocoustic behaviour of the propellers. The aerodynamic measurements show that the bio-inspired propeller can provide the same thrust as the baseline propeller under the same power input in hover condition, but the rotational regime was lower, indicating higher lift coefficient for the bio-inspired blade. However, the reduction in noise is up to 4 $\mathrm{dB}$ and can be ascribed to the small force variation of the new blade. Thrust standard deviation $\sigma_{T}$, representative of force oscillation, is observed to be $24 \%$ lower than the baseline propeller. As seen in $\S 2.1$, loading noise is related to force variation. Furthermore, the bio-inspired propeller generated a smaller wake region and demonstrated a faster decay rate in tip vortex strength.

Another noise reduction strategy inspired by nature is presented in [73]: by mimicking the idowny coat of the barn owl to reduce the noise generated by an airfoil. A numerical investigation was carried out on an airfoil with finlet fences. The simulation was carried out on a baseline geometry 


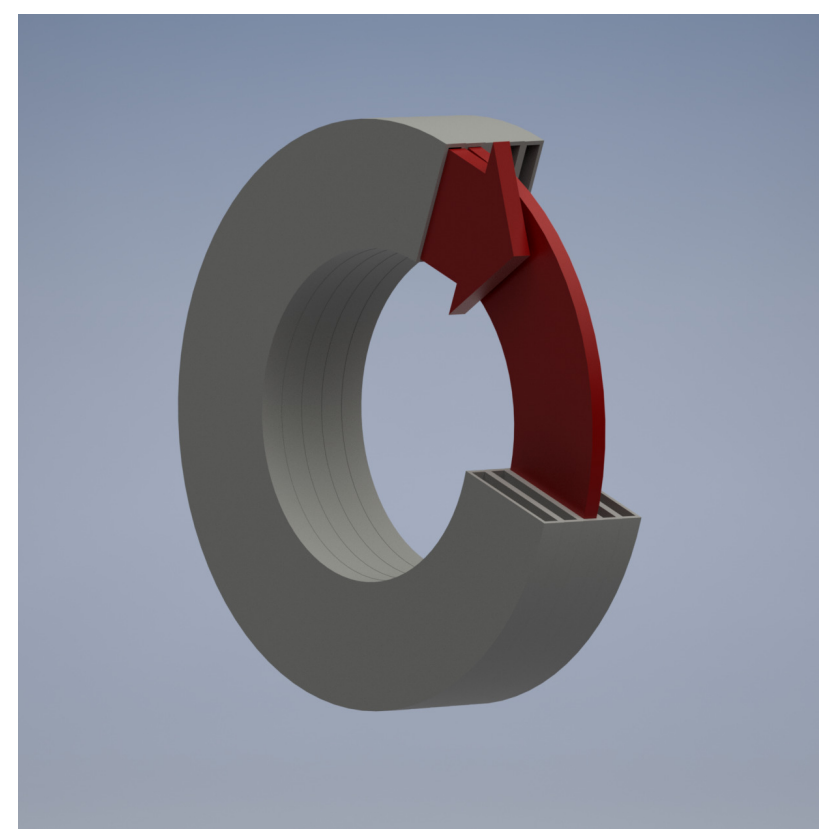

Figure 7. 3D Rendering of the proposed configuration of the Ultra-Open Metamaterial.

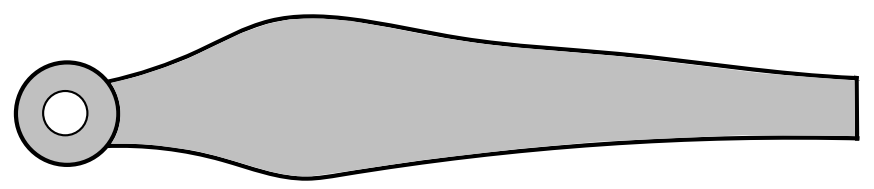

(a)

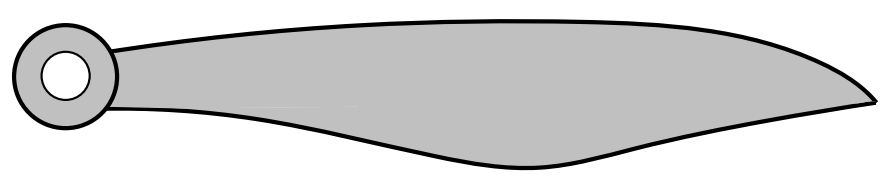

(b)

Figure 8. Representation of the considered baseline blade (a) and of the new bio-inspired blade (b).

and later the finlets were added. The study was performed by using an implicit large eddy model. The comparison between $c_{f}$ and $c_{P}$ for the baseline and the owl-inspired geometry shows that the add-on does not significantly degrade the aerodynamic performance. Furthermore, spectral analysis shows a slight reduction in pressure spectra at high frequencies near the TE of the airfoil. At the present state-of-art, this technology has not been tested on a propeller, so it would be interesting to investigate this innovative geometry both numerical and experimental in order to understand if it is really applicable to drone propellers.

\section{Conclusion}

In this paper the focus is on the noise generated by small rotors, the aim being to identify which passive noise control strategies can be employed on a drone propeller. The main noise sources for this application concern the interaction between the BL and the TE of the blade. This paper presents several techniques to control this noise source. Even though, noise control is the main focus, aerodynamic performance is also taken in count in order to guarantee the success of the mission.

The first strategy to reduce noise emission was to employ an optimized geometry by taking into account acoustic constraints in the multi-disciplinary optimization process. These solutions led to 
blade geometry that reduce noise for a specific operating configuration, so it is not sure that in other configurations the behaviour would be exactly the same, both in terms of thrust and noise generation. The effect of chord distribution and of pitch angle was analyzed, indicating significant noise reduction, but the drawback was a loss of aerodynamic features.

By taking ideas from nature, in particular the owl wing, innovative blade geometries may be employed, the most effective, seemingly,being the application of serration. The most investigated configuration is the sawtooth pattern at the TE, which has been shown to reduce the broad-band noise component. From a theoretical point of view, this effect can be related to a reduction in coherent structures in the pressure field. This assumption is confirmed by statistical analysis, which shows lower PDF tails when serration is employed at the TE. Another configuration involves the use of fractal serration, the effect, even for this configuration, being related to the interaction between the coherent structures and the serration.

Another strategy to reduce TE noise is the use of porous materials. The effect of porosity on rotor noise has been studied principally for wind turbines but it seems very interesting also for UAV rotors. In fact, it has been proved that porosity produces a reduction in turbulence length scale. The next step is to employ this technology on a small-scale propeller and test it. Furthermore, metamaterials can be designed as highly efficient sound barriers for a target frequency. The development in metamaterials science may, in a few years, lead to the realization of a ducted propeller with specific sound characteristics.

On the other hand, in order to reduce the tonal noise component, a boundary layer tripping system can be applied on the suction side of the propeller blade in the form of a simple adhesive aluminium strip. By using this system, laminar to turbulent transition is forced at $5 \%$ of the chord. This effect results in broad-band noise radiating from the TE in the high frequency region and seems to have no effect on thrust generation; rather it should reduce drag force since the efficiency of the propeller should increase.

Finally, this paper presented an innovative type of geometry inspired by nature. This particular geometry mimics the planform shape of cicada wings and maple seeds. The experimental results show that the bio-inspired wing can provide the same thrust as a baseline propeller. Additionally, a reduction in loading noise was observed and can be attributed to the reduction in standard thrust deviation.

\section{References}

1. Floreano, D.; Wood, R.J. Science, technology and the future of small autonomous drones. Nature 2015, 521,460-466. doi:10.1038/nature14542.

2. Tauro, F.; Porfiri, M.; Grimaldi, S. Surface flow measurements from drones. Journal of Hydrology 2016, 540, 240-245. doi:10.1016/j.jhydrol.2016.06.012.

3. Bandini, F.; Jakobsen, J.; Olesen, D.; Reyna-Gutierrez, J.A.; Bauer-Gottwein, P. Measuring water level in rivers and lakes from lightweight Unmanned Aerial Vehicles. Journal of Hydrology 2017, 548, 237-250.

4. Venturi, S.; Di Francesco, S.; Materazzi, F.; Manciola, P. Unmanned aerial vehicles and Geographical Information System integrated analysis of vegetation in Trasimeno Lake, Italy. Lakes and Reservoirs: Research and Management 2016, 21, 5-19. doi:10.1111/lre.12117.

5. Agency, E.A.S. Introduction of a regulatory framework for the operation of unmanned aircraft systems in the 'open' and 'specific' categories. Opinion No 01/2018 2018.

Cherney, M. Delivery Drones Cheer Shoppers, Annoy Neighbors, Scare Dogs. Wall Street Journal 2018.

7. Morrell, S.; Taylor, R.; Lyle, D. A review of health effects of aircraft noise. Australian and New Zealand Journal of Public Health 1997, 21, 221-236. doi:10.1111/j.1467-842X.1997.tb01690.x.

8. Giones, F.; Brem, A. From toys to tools: The co-evolution of technological and entrepreneurial developments in the drone industry. Business Horizons 2017, 60, 875-884. doi:10.1016/j.bushor.2017.08.001. 
9. Mulero-Pázmány, M.; Jenni-Eiermann, S.; Strebel, N.; Sattler, T.; Negro, J.J.; Tablado, Z. Unmanned aircraft systems as a new source of disturbance for wildlife: A systematic review. PLOS ONE 2017, 12, 1-14. doi:10.1371/journal.pone.0178448.

10. Ditmer, M.A.; Vincent, J.B.; Werden, L.K.; Tanner, J.C.; Laske, T.G.; Iaizzo, P.A.; Garshelis, D.L.; Fieberg, J.R. Bears Show a Physiological but Limited Behavioral Response to Unmanned Aerial Vehicles. Current Biology 2015, 25, 2278-2283. doi:10.1016/j.cub.2015.07.024.

11. Kempf, N.; Hüppop, O. What effect do airplanes have on birds?-a summary and update of "Wie wirken Flugzeuge auf Vögel?, Naturschutz und Lanschaftsplanung, 30-1 (17-28). 1998”[Internet]. 1998, 1998.

12. Christiansen, F.; Rojano-Doñate, L.; Madsen, P.T.; Bejder, L. Noise Levels of Multi-Rotor Unmanned Aerial Vehicles with Implications for Potential Underwater Impacts on Marine Mammals. Frontiers in Marine Science 2016, 3, 1-9. doi:10.3389/fmars.2016.00277.

13. Smith, C.E.; Sykora-bodie, S.T.; Bloodworth, B.; Pack, S.M.; Spradlin, T.R.; Leboeuf, N.R. Assessment of known impacts of unmanned aerial systems (UAS) on marine mammals: data gaps and recommendations for researchers in the United States. Journal of Unmanned Vehicle Systems 2016, 14, 1-14.

14. Cannard, M.; Joseph, P.; Kim, J.W.; Paruchuri, C.C. Slitted leading-edge profiles for the reduction of broadband interaction noise; physical mechanisms and performance. 25th AIAA/CEAS Aeroacoustics Conference, 2019, p. 2511. doi:10.2514/6.2019-2511.

15. Hasheminejad, S.M.; Chong, T.P.; Joseph, P.; Lacagnina, G. Effect of Leading-Edge Serrations on Trailing-Edge-Bluntness Vortex-Shedding Noise Radiation. 25th AIAA/CEAS Aeroacoustics Conference, 2019, p. 2437. doi:10.2514/6.2019-2437.

16. Serré, R.; Fournier, H.; Moschetta, J.M. A design methodology for quiet and long endurance MAV rotors. International Journal of Micro Air Vehicles 2019, 11, 1756829319845937. doi:10.1177/1756829319845937.

17. Pagliaroli, T.; Camussi, R.; Candeloro, P.; Giannini, O.; Bella, G.; Panciroli, R. Aeroacoustic Study of small scale Rotors for mini Drone Propulsion: Serrated Trailing Edge Effect. 2018 AIAA/CEAS Aeroacoustics Conference 2018. doi:10.2514/6.2018-3449.

18. Gur, O.; Rosen, A. Optimizing Electric Propulsion Systems for Unmanned Aerial Vehicles. Journal of Aircraft 2009, 46, 1340-1353. doi:10.2514/1.41027.

19. Pagliaroli, T.; Moschetta, J.m.; Benard, E.; Nana, C. Noise signature of a MAV rotor in hover. 49th International Symposium of Applied Aerodynamics Lille, 24-25-26 March 2014 2014, pp. 24-25.

20. Sinibaldi, G.; Marino, L. Experimental analysis on the noise of propellers for small UAV. Applied Acoustics 2013. doi:10.1016/j.apacoust.2012.06.011.

21. Gur, O.; Rosen, A. Design of a Quiet Propeller for an Electric Mini, 2009. doi:10.2514/1.38814.

22. JanakiRam, D.; Scruggs, B. Investigation of performance, noise and detectability characteristics of small-scale remotely piloted vehicle /RPV/ propellers. 7th Aeroacoustics Conference 1981, 19, 1052-1060. doi:doi:10.2514/6.1981-2005.

23. Serré, R.; Chapin, V.; Moschetta, J.M.; Fournier, H. Reducing the noise of Micro-Air Vehicles in hover. International Micro Air Vehicle Conference and Flight Competition 2017, pp. 51-59.

24. Leslie, A.; Wong, K.C.; Auld, D. Broadband Noise Reduction on a mini-UAV Propeller. 14th AIAA/CEAS Aeroacoustics Conference (29th AIAA Aeroacoustics Conference), 2008. doi:10.2514/6.2008-3069.

25. Nelson, P.A.; Morfey, C.L. Aerodynamic Sound Production. Journal of Sound and Vibration 1981, 79, $263-289$. 26. Rozenberg, Y.; Roger, M.; Moreau, S. Rotating Blade Trailing-Edge Noise: Experimental Validation of Analytical Model. AIAA Journal 2010. doi:10.2514/1.43840.

27. Intravartolo, N.; Sorrells, T.; Ashkharian, N.; Kim, R. Attenuation of Vortex Noise Generated by UAV Propellers at Low Reynolds Numbers. 55th AIAA Aerospace Sciences Meeting, 2017. doi:10.2514/6.2017-2019.

28. Farassat, F.; Succi, G.P. A review of propeller discrete frequency noise prediction technology with emphasis on two current methods for time domain calculations. Topics in Catalysis 1980. doi:10.1016/0022-460X(80)90422-8.

29. Zawodny, Nikolas S., B.; Burley, Casey L.. Acoustic Characterization and Prediction of Representative, Small-Scale Rotary-Wing Unmanned Aircraft System Components . 72nd American Helicopter Society (AHS) Annual Forum; 17-19 May 2016; West Palm Beach, FL; United States 2016.

30. Zawodny, N.S.; Boyd Jr, D.D. Investigation of rotor-airframe interaction noise associated with small-scale rotary-wing unmanned aircraft systems. American Helicopter Society Paper 2017. 
31. Ffowcs Williams, J. E., and Hawkings, D.L. Sound Generation by Turbulence and Surfaces in Arbritary Motion . Philosophical Transactions of the Royal Society of London. Serie A, Mathematical and Physical Sciences 1969, 264, 321-342. doi:10.1098/rsta.1969.0031.

32. Succi, G.P. Design of Quiet Efficient Propellers. SAE Technical Paper. SAE International, 1979, p. 14. doi:10.4271/790584

33. Schlinker, R.H.; Amiet, R.K. Helicopter Rotor Trailing Edge Noise. 7th Aeroacoustics Conference 1981, p. 2001. doi:10.2514/6.1981-2001.

34. Rozenberg, Y.; Roger, M.; Moreau, S.; Division, D.; Cedex, C.; Systems, V.T.; Normand, L.; Verri, L. Fan Blade Trailing-Edge Noise Prediction Using RANS Simulations. Journal of the Acoustical Society of America 2008, 123, 5207-5212. doi:10.2514/6.2010-3720.

35. Corcos, G. The structure of the turbulent pressure field in boundary-layer flows. Journal of Fluid Mechanics 1964, 18, 353-378. doi:10.1017/S002211206400026X.

36. Pang, E.; Cambray, A.; Rezgui, D.; Azarpeyvand, M.; Showkat Ali, S.A. Investigation Towards a Better Understanding of Noise Generation from UAV Propellers. 2018 AIAA/CEAS Aeroacoustics Conference, 2018. doi:10.2514/6.2018-3450.

37. Betz, A. Schraubenpropeller mit geringstem Energieverlust. Mit einem Zusatz von 1. Prandtl. Nachrichten von der Gesellschaft der Wissenschaften zu Göttingen, Mathematisch-Physikalische Klasse 1919, 1919, 193-217.

38. Patrick, H.; Finn, R.W.; Stich, C.K. Two and Three-Bladed Propeller Design For the Reduction of Radiated Noise. 3rd AIAA/CEAS Aeroacoustics Conference 1997, pp. 934-950. doi:10.2514/6.1997-1710.

39. Roncz, J.G. Propeller Development for the Rutan Voyager. Technical report, SAE Technical Paper, 2018. doi:10.4271/891034

40. Adkins, C.N.; Liebeckt, R.H. Design of Optimum Propellers. Journal of Propulsion and Power 1994, 10, 676-682. doi:10.2514/3.23779.

41. Sobieszczanski-sobieski, J.; Haftka, R.T. Multidisciplinary aerospace design optimization - Survey of recent developments. Structural optimization 1996, 14, 1-23.

42. Gur, O.; Rosen, A. Optimization of Propeller Based Propulsion System. Journal of Aircraft 2009, 46, 95-106. doi:10.2514/1.36055.

43. Gur, O.; Rosen, A. Multidisciplinary Design Optimization of a Quiet Propeller. 14th AIAA/CEAS Aeroacoustics Conference (29th AIAA Aeroacoustics Conference) 2008, 3073, 5-7. doi:10.2514/6.2008-3073.

44. Clark, I.A.; Daly, C.A.; Devenport, W.; Alexander, W.N.; Peake, N.; Jaworski, J.W.; Glegg, S. Bio-inspired canopies for the reduction of roughness noise. Journal of Sound and Vibration 2016. doi:10.1016/j.jsv.2016.08.027.

45. Peake, N. The aeroacoustics of the Owl. Lecture Notes in Mechanical Engineering, 2016. doi:10.1007/978-3-662-48868-3_2.

46. Jaworski, J.W.; Peake, N. Aerodynamic noise from a poroelastic edge with implications for the silent flight of owls. Journal of Fluid Mechanics 2013, [arXiv:1011.1669v3]. doi:10.1017/jfm.2013.139.

47. Chong, T.P.; Vathylakis, A. On the aeroacoustic and flow structures developed on a flat plate with a serrated sawtooth trailing edge. Journal of Sound and Vibration 2015. doi:10.1016/j.jsv.2015.05.019.

48. Avallone, F.; Van Der Velden, W.C.; Ragni, D.; Casalino, D. Noise reduction mechanisms of sawtooth and combed-sawtooth trailing-edge serrations. Journal of Fluid Mechanics 2018. doi:10.1017/jfm.2018.377.

49. Howe, M.S. Noise produced by a sawtooth trailing edge. The Journal of the Acoustical Society of America 1991, 90, 482-487. doi:10.1121/1.401273.

50. Ning, Z.; Hu, H. An Experimental Study on the Aerodynamics and Aeroacoustic Characteristics of Small Propellers. 54th AIAA Aerospace Sciences Meeting, 2016. doi:10.2514/6.2016-1785.

51. Hasheminejad, S.M.; Chong, T.P.; Joseph, P.; Lacagnina, G. Airfoil Self-Noise Reduction Using Fractal-Serrated Trailing Edge. 2018 AIAA/CEAS Aeroacoustics Conference, 2018. doi:10.2514/6.2018-3132.

52. Ragni, D.; Avallone, F.; van der Velden, W.C.; Casalino, D. Measurements of near-wall pressure fluctuations for trailing-edge serrations and slits. Experiments in Fluids 2019, 60, 0. doi:10.1007/s00348-018-2654-5.

53. Arce León, C.; Merino-Martínez, R.; Ragni, D.; Avallone, F.; Snellen, M. Boundary layer characterization and acoustic measurements of flow-aligned trailing edge serrations. Experiments in Fluids 2016, 57. doi:10.1007/s00348-016-2272-z. 
54. Leslie, A.; Wong, C.; Auld, D. Experimental analysis of the radiated noise from a small propeller. Proceedings of 20th International Congress on Acoustics, ICA 2010.

55. McAlpine, A.; Nash, E.; Lowson, M. On the generation of discrete frequency tones by the flow around an aerofoil. Journal of Sound and Vibration 1999, 222, 753-779.

56. Graham, L.R.R. The Silent FLight of Owl. The Aeronautical Journal 1934, 38, 837-843. doi:10.1017/S0368393100109915.

57. Lee, S. Reduction of Blade-Vortex Interaction Noise Through Porous Leading Edge. AIAA Journal 1994, 32, 480-488. doi:doi.org/10.2514/3.12011.

58. Revell, J.D. Trailing-Edge Flap Noise Reduction by Porous Acoustic Treatment. 3rd AIAA/CEAS Aeroacoustic Conference 1997, pp. 493-505. doi:10.2514/6.1997-1646.

59. Search, H.; Journals, C.; Contact, A.; Iopscience, M.; Dyn, F.; Address, I.P. Application of porous material to reduce aerodynamic sound from bluff bodies. Fluid Dynamics Research 2010, 015004. doi:10.1088/0169-5983/42/1/015004.

60. Geyer, T.; Sarradj, E.; Fritzsche, C. Porous airfoils : noise reduction and boundary layer effects. International Journal of Aeroacoustics 2010, 9, 787-820. doi:10.1260/1475-472X.9.6.787.

61. Sarradj, E.; Geyer, T. Noise Generation by Porous Airfoils. 13th AIAA/CEAS Aeroacoustics Conference (28th aeroacoustic conference) 2007. doi:10.2514/6.2007-3719.

62. Jiang, C.; Moreau, D.; Yauwenas, Y.; Fischer, J.R.; Doolan, C.J.; Gao, J.; Jiang, W.; McKay, R.; Kingan, M. Control of rotor trailing edge noise using porous additively manufactured blades. 2018 AIAA/CEAS Aeroacoustics Conference, 2018. doi:10.2514/6.2018-3792.

63. Moreau, S.; Dignou, B.; Jaiswal, P.; Yakhina, G.R.; Pasco, Y.; Sanjose, M.; Alstrom, B.; Atalla, N. Trailing-edge noise of a flat plate with several liner-type porous appendices. 2018 AIAA/CEAS Aeroacoustics Conference, 2018. doi:10.2514/6.2018-3119.

64. Rubio Carpio, A.; Merino Martínez, R.; Avallone, F.; Ragni, D.; Snellen, M.; van der Zwaag, S. Experimental characterization of the turbulent boundary layer over a porous trailing edge for noise abatement. Journal of Sound and Vibration 2019, 443, 537-558. doi:10.1016/j.jsv.2018.12.010.

65. Showkat Ali, S.A.; Azarpeyvand, M.; Ilario da Silva, C.R. Experimental Study of Porous Treatments for Aerodynamic and Aeroacoustic Purposes. 23rd AIAA/CEAS Aeroacoustics Conference 2017, p. 3358. doi:10.2514/6.2017-3358.

66. Applications, T.e. Specification of Porous Materials for Low-Noise. 24th AIAA/CEAS Aeroacoustic Conference 2014, pp. 1-19. doi:10.2514/6.2014-3041.

67. Joseph, P.F. Poro-Serrated Trailing-Edge Devices for Airfoil Self-Noise. AIAA Journal $2015,53$. doi:10.2514/1.J053983.

68. Chong, T.P.; Dubois, E.; Vathylakis, A. Aeroacoustic and flow assessments of the poro-serrated trailing edges. 22nd AIAA/CEAS Aeroacoustics Conference, 2016. doi:10.2514/6.2016-2833.

69. Chong, T.P.; Dubois, E. Optimization of the poro-serrated trailing edges for airfoil broadband noise reduction. The Journal of the Acoustical Society of America 2016, 140, 1361-1373. doi:10.1121/1.4961362.

70. Ghaffarivardavagh, R.; Nikolajczyk, J.; Anderson, S.; Zhang, X. Ultra-open acoustic metamaterial silencer based on Fano-like interference. Physical Review B 2019, 99, 1-10. doi:10.1103/PhysRevB.99.024302.

71. Újsághy, O.; Kroha, J.; Szunyogh, L.; Zawadowski, A. Theory of the Fano resonance in the STM tunneling density of states due to a single Kondo impurity. Physical review letters 2000, 85, 2557.

72. Ning, Z.; Hu, H. An Experimental Study on the Aerodynamic and Aeroacoustic Performances of a Bio-Inspired UAV Propeller. 35th AIAA Applied Aerodynamics Conference, 2017. doi:10.2514/6.2017-3747.

73. Bodling, A.; Agrawal, B.R.; Sharma, A.; Clark, I.; Alexander, W.N.; Devenport, W.J. Numerical Investigations of Bio-Inspired Blade Designs to Reduce Broadband Noise in Aircraft Engines and Wind Turbines. 55th AIAA Aerospace Sciences Meeting, 2017, p. 0458. doi:10.2514/6.2017-0458.

Acknowledgments: This work was supported by the Italian Ministry of Education, University and Research under the PRIN grant No.20154EHYW9 "Combined numerical and experimental methodology for fluid structure interaction in free surface flows under impulsive loading". 